Polarization interferometers: applications in microscopy and macroscopy. By $M$. Françon and S. MaLlick. Pp. 159. London: Wiley, 1971. Price $£ 4 \cdot 50$.

Polarization interferometers are ones in which the two coherent beams required for interference are produced from a single source by means of a birefringent beam-splitter, i.e. use is made of the separation of the $E$ and $O$ rays. The two resulting beams are necessarily poiarized at right angles, so there must be some arrangement for making their planes of polarization parallel again before interference can take place. There are many combinations of ways of carrying out these functions, and polarization interferometers can be employed for all the usual applications of interferometers. The field is therefore large and complicated, and the present monograph by Françon and Mallick aims to survey the possibilities and to bring together information otherwise only available from scattered sources. Their stated aim is to minimize the mathematics, and to concentrate on physical principles and on help with the actual use of the instruments. There are thus chapters on polarzation in general, and on birefringent beam-splitters and other components, followed by several chapters on typical applications, such as the observation of phase objects, the measurement of optical thickness, the testing of optical systems, and on polarization methods in interference microscopy. The authors are well qualified to survey this field as they are from the Institute $\mathrm{d}^{\prime} \mathrm{Optique}$ in Paris, where much of the work has been done, and they themselves have originated many of the instruments and variants described.

However, having said all this, the book itself is a little disappointing; the approach seems to be academic rather than practical, and the result is a textbook rather than a handbook. We are promised practical advice on how to set up the instruments, and in a few cases some useful component parameters are given: however, there is usually no such information, and the book would be much more helpful if we could have the benefit of the authors' considerable experience. Also, the diagrams of the instruments are conventionally schematic rather than practical, and in most cases give little idea as to what one should actually do. It would be useful to have more illustrations of the appearance of the fringes obtained; there are, in all, seven photographs of interference effects and about the same number of schematic diagrams of them, but usually there is nothing against which to confirm ones observations, or to assist in correcting maladjustments. A possibly more serious omission is that there is little indication of the advantage of using interferometers involving polarization, to be set against the fact that polarization components such as Savart plates and Wollaston prisms are very expensive. It is mentioned briefly in the introduction that polarization interferometers are simple to use, and that they are stable because the beam separation is small; two cases are described (differential interference microscopy and coherence measurement) in which a small separation is actually required and can conveniently be obtained by polarization. In the wide-angle Michelson interferometer a large entrance cone is achieved by making use of the reduced change of path difference with angle when a polarizing beam-splitter is used; however, one has to search for these cases in which some advantage is claimed, and even in these the results can be achieved by other means. It becomes evident from the many examples given that one can make polarization versions of interferometers to cover any of the usual applications - but it remains far from clear why one should do so, how much advantage is to be gained, and whether there are things that cannot be done any other way.

As regards completeness, one is of course bound to be able to find omissions from any compilation of this sort, but there are nevertheless a few rather surprising ones. For example, the Savart polariscope is described, and some variants of it, but there is no mention of the considerable improvements in sensitivity achieved - in Paris - in the version devised by Lyot and later improved by Dollfus. There is no mention of Sinton's polarization interferometer, or of the polarizationinterferencespectrometerdevised by Mertz, which is surely original enough to merit inclusion.

As stated in the introduction, theory was kept to a minimum in the body of the book, and it was a good idea to put some of the detailed theoretical discussions in appendices at the end. A minor fault here is probably a matter of editing - a formula used on p. 19 is said to be derived in Appendix l, but the resulting expression is given there in a slightly different form, giving the reader the exercise of showing it to be equi- valent; furthermore, a different symbol is used for the important angle it contains, which might cause some confusion.

On the whole, this book is useful in that it collects together information about some ingenious optical instruments which are probably not well enough known, and it may thereby encourage the reader to investigate further their undoubtedly useful properties.

Physics Department

J. E. GEAKE

UMIST

Manchester M60 1OD

England

\section{Principles and Practice of} $X$-ray Spectrometric Analysis. By Eugene P. Bertin. Pp. xxiv +679. New York: Plenum Press, 1970. Price $£ 17.50$, DM 150.00 .

The content of this book is an important attempt to fulfil the aims defined in the title. According to its author it is primarily intended for students and technicians either having no practical experience of $X$-ray spectrometric analysis or having some practical experience of the method but not enough formal instruction in its principles.

It is evident that sound basic knowledge of $X$-ray physics in general and spectroscopy in particular is implicitly involved throughout this text-book. The first two chapters (about one tenth of the whole) are explicitly devoted to a summary of the laws; the point of view is kept elementary, with little up-to-date theoretical justification. However, it should encourage the reader to take an interest in the fundamental aspects of the subject and to undertake more academic reading. It is mainly intended as a text-book for users of commercially available $X$-ray fluorescence spectrometers; as such, it will offer them a very valuable tool. Accordingly chapter 3 and the beginning of chapter 4 deal with $X$-ray secondary excitation. One also finds information on essential pieces of equipment (X-ray tubes, collimators, flat and curved crystal spectrometers, analyser crystals and multilayer films, various detectors, etc.), and how to use them appropriately. Non-dispersive analysis and (briefly in the last chapter) electron-probe microanalysis are also mentioned.

Four chapters of practical importance are dedicated to qualitative and semi- 
quantitative analysis and 'performance criteria' of the method, referring to the usual criteria of any analytical method; special reference is made to some specific problems of X-ray spectrometric anal$y$ sis such as 'matrix effects'. The next two chapters deal with basic methods leading to actual quantitative results, standards and calibration and special emphasis is given to corrections for absorption enhancement effects.

Useful practical, though not exhaustive, information about the choice of specimen, preparation methods and their consequences will be found in the next two chapters; attention is often drawn to certain problems of fundamental importance like surface state and granulometry which, unfortunately, cannot be given their full importance in the framework of such a chapter. The remaining chapters describe what the author terms 'unconventional modes of operation' of the spectrometer and 'related X-ray methods' of analysis. In fact one chapter deals with the analysis of films and platings, the next with small specimens and $X$-ray probe techniques, while some information is provided on $X$-ray absorption spectrometry with mention of scattering techniques, in so far as these methods can be taken care of by means of commercial equipment. Photo- and Auger electron spectroscopy is also described.

Incidentally, this text-book, once more, raises implicitly the practical problems of notation and of systematic and numerical values in $\mathrm{X}$-ray spectroscopy and diffraction.

Unfortunately physico-chemical effects on X-ray spectra are hardly mentioned; the old view of 'inner orbitals substantially unaffected by valence' is taken for granted and solid-state physics implications practically discarded.

The bibliography for such a broad domain connected with fundamental aspects of radiation and matter and covering so many fields of application cannot be exhaustive; in particular, references to some important European publications and to modern books of fundamental interest are lacking.

Such as it is, well printed with clear figures and tables, this rather big book represents an important contribution to the field and will be very helpful to users of $X$-ray spectrometry which has become a wide-spread and competitive analytical method.

Y. CAUCHOIS

Laboratoire de Chimie Physique

11 , Rue P. et M. Curie

75-Paris Ve

France

\section{Books Received}

The following books have been received by the Editor. Brief and generally uncritical notices are given of works of marginal crystallographic interest; occasionally a book of fundamental interest is included under this heading because of difficulty in finding a suitable reviewer without great delay.

Optical microscopy for the materials sciences. By JAMES H. Richardson. Pp. $x+692$. New York: Marcel Dekker, 1971. Price $\$ 29.50$.

This book brings together in one volume a comprehensive discussion of modern optical microscopy and photomicroscopy necessary to prepare and examine specimens of a broad range of materials.

The methods for preparing specimens of material for optical microscopes that use transmitted or incident light are described. Also included here are extensive tables that give specific solutions for chemical and electrolytic polishing and etching of incident-light specimens for a large number of materials. A subsequent chapter is devoted to the techniques used in the qualitative and quantitative measurements of a specimen under microscopic examination. Other topics explored in this volume include: photomicrography and photomacrography, laboratory safety, and microscope accessories.

The book is prepared by lithography directly from typed material and the effect is very clear and quite pleasing to the eye. There are numerous figures of very high quality.

\section{Handbook of electronic ma- terials. Vol.6. Silicon nitride for microelectronic appli- cations, Part 2. BY JoHN T. Milek. Pp. vii +117. New York: Plenum Press, 1972. Price $\$ 14.00$.}

Contents : Introduction ; Diffusion mask applications ; Glass-to-Metal seals ; Passivation applications; Isolation; Memory devices; Capaciiors; Radiation hardening applications; Miscellaneous devices and applications; References. 